\title{
Paired-associate latencies after the last error'
}

Lloyd R. Peterson

INDIANA UNIVERSITY

\begin{abstract}
Latencies in paired-associate learning were examined for 10 trials beginning after the last error on an individual pair. Latencies decreased over the first eight of these trials, the largest decrease occurring from the first to the second trial after the last error.

\section{Problem}

For an incremental view of learning the latency of response would be expected to decrease after the $S$ stops making errors. However, decreasing latencies after errors cease are difficult to incorporate into an all-ornone model of learning. Millward (1964) found decreasing latencies in a two-alternative button-pressing task for several trials after the last error. However, the guessing probability was high with only two responses, and an allor-none interpretation could assume that the $\mathrm{S}$ was right by guessing for some of the pairs in the early trials after the last error. The decreasing latencies could then be attributed to learning of these pairs. The present study utilized a larger number of alternatives, reducing the guessing probability.
\end{abstract}

\section{Method}

Fifteen undergraduates at Indiana University were paid to serve as Ss. Each was given 20 trials on a list of 10 paired-associates presented on a memory drum. An $\mathrm{S}$ was permitted as much time as necessary to make a response during the anticipation period when a cue was presented alone. The duration of this interval was recorded on a Standard electric timer. The drum motor, having been stopped during this period, was started again when the $\mathrm{S}$ spoke into a microphone connected to a noise-actuated relay. Tape recordings were made to check the accuracy of the E's recording. Not included among the 15 Ss were three individuals who failed to speak consistently with sufficient vigor to operate the relay.

The stimuli were 10 consonant bigrams, no two of which had any letters in common. Responses were the digits 1 to 8 . Two of the responses were paired with two different bigrams in an attempt to minimize the use of guessing strategies. Ten orders of the list were used with each S. There was an 8-sec. rest interval between trials.

\section{Results}

The latencies of the responses, beginning with the first trial after the last error on an individual pair, are graphed in Fig. 1. Only pairs for which 10 trials beyond the last error were available were used in the analysis. A median latency for each $\mathrm{S}$ was obtained. Means of the Ss' medians were then computed to give

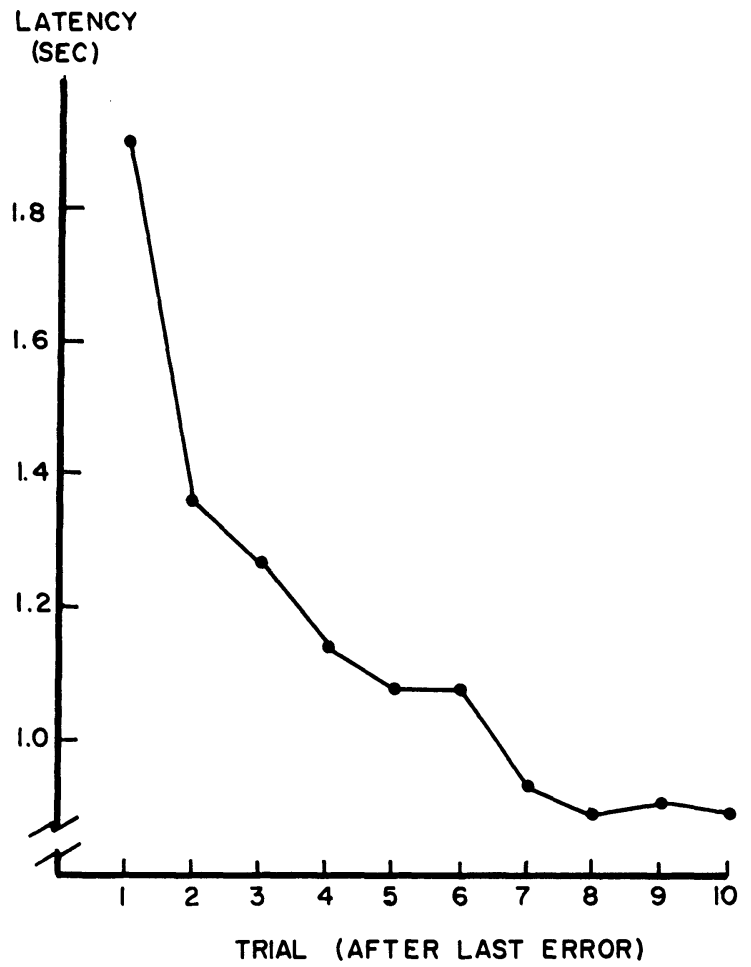

Fig. 1. Mean latency for 10 trials after the last error in a pairedassociate task.

the decreasing curve of Fig. 1. An analysis of variance of the medians yielded an F of 27.9, significant at the .001 level. The data show decreasing latencies through eight trials after the last error.

No reliable trends in latency prior to the last error were found. Mean proportions correct, averaging over seven trials before the last error and including pairs for which there were six consecutive correct anticipations, were .14, .18, .32, .29, .49, .35, and .43. Recall frequencies for individual pairs increased before the last error.

\section{Diseussion}

There are selection problems in interpreting data before the last error. Fewer Ss and pairs are represented in the early trials of the above frequency data than in the later trials. It might at least be said that there is evidence of recall above the guessing level (.125) before occurrence of the last error. 
The data after the last error seem unequivocal. The continued decrease in latency over a sequence of trials could not be attributed to replacement of guesses by learned recalls. The guessing probability is too low.

It is clear that the ability of the $S$ to respond correctly is a function of the length of time allowed for him to respond. In a learning experiment with fixed anticipation interval, relative frequencies of correct responses will not be independent of latency. The decision of the $\mathrm{E}$ as to when an $S$ has formed an association under such conditions is arbitrary and difficult to reconcile with an assumption that associations are formed in an all-ornone manner.

A reasonable integration of frequency and latency data may require a distinction between implicit occurrence of a response and the S's decision to vocalize the response. The frequency data in an experiment where unlimited time is given for recall may reflect the S's ability to recognize the response that is correct when itoccurs to him, as well as the liklihood that he thinks of the correct response. The latency data after the last error would then reflect speed of occurrence of the correct response after the $\mathrm{S}$ has learned to recognize it when it occurs. Speed of occurrence appears to be a measure of strength of an association which changes gradually over trials. Recognition of what is correct or erroneous does not seem to be entirely dependent on speed of occurrence. The failure to find systematic changes in latency before the last error. (Cf. Millward, 1964) may in part be due to interference effects from erroneous responding (Peterson \& Brewer, 1963).
References

MILLWARD, R. Latency in a modified paired-associate learning experiment. J. verbal Learn. verbal Behav., 1964, 3, 309-316. PETERSON, L. R., \& BREWER, C. L. Confirmation, correction, and contiguity. J. verbal Learn. verbal Behav., 1963, 1, 365-371.

Note

1. This research was facilitated by National Science Foundation Grant GB 704.

\section{Erratum}

JENKINS, J。 J., \& FOSS, D. J. An experimental analysis of pseudomediation. Psychon. Sci., 1965, 2, 99-100. The Editor regrets that, due to an error in his office, Table 1 (referred to near the top of column 1 on page 100) was omitted from the article. It is reproduced below.

Table 1. Means and Pairwise t-Tests of Criterion Measures for Each Learning Stage

\begin{tabular}{|c|c|c|c|c|c|}
\hline Stage & Group & $\mathrm{NC}$ & $C-1$ & $\mathrm{C}-2$ & $\mathrm{C}-2 \mathrm{~s}$ \\
\hline \multirow{3}{*}{ I } & Experimental & 10.55 & 13.55 & 15.60 & 16.05 \\
\hline & Control & 12.55 & 12.35 & 14.85 & 16.10 \\
\hline & t-value & $2.58^{*}$ & 1.28 & .78 & .05 \\
\hline \multirow{3}{*}{ II } & Experimental & 13.35 & 10.25 & 12.10 & 13.00 \\
\hline & Control & 19.30 & 7.30 & 9.40 & 10.95 \\
\hline & t-value & $6.72 * *$ & $6.08 * *$ & $4.67 * *$ & $2.76 * *$ \\
\hline \multirow{3}{*}{ III } & Experimental & 15.70 & 9.25 & 10.95 & 11.75 \\
\hline & Control & 15.35 & 9.55 & 12.25 & 14.00 \\
\hline & $\mathrm{t}$-value & .34 & .47 & 1.92 & $2.92 * *$ \\
\hline
\end{tabular}

* Significant at .05 level

** Significant at .01 level 